\title{
La formation des enseignants dans un pays fédéral
}

Le cas de la République d'Argentine

Teacher training in a federal country. The case of the Republic of Argentina La formación de los profesores en un país federal. El caso de la República de Argentina

\section{Beatriz Alen}

Traducteur : Philippe Rabaté

\section{OpenEdition} Journals

Édition électronique

URL : http://journals.openedition.org/ries/941

DOI : 10.4000/ries.941

ISSN : 2261-4265

\section{Éditeur}

Centre international d'études pédagogiques

\section{Édition imprimée}

Date de publication : 1 décembre 2010

Pagination : 73-82

ISBN : 978-2-8542-583-7

ISSN : $1254-4590$

\section{Référence électronique}

Beatriz Alen, «La formation des enseignants dans un pays fédéral », Revue internationale d'éducation de Sèvres [En ligne], 55 | décembre 2010, mis en ligne le 01 décembre 2013, consulté le 03 mai 2019. URL : http://journals.openedition.org/ries/941 ; DOI : 10.4000/ries.941 


\section{La formation des enseignants dans un pays fédéral*}

\section{Le cas de la République d'Argentine}

\section{Beatriz Alen}

La nouvelle étape que connaît actuellement le système éducatif argentin se caractérise par deux grandes politiques: d'une part, la démocratisation du système - extension du caractère obligatoire de l'école jusqu'au niveau secondaire, élargissement de la couverture et hiérarchisation des processus pédagogiques et, d'autre part, l'amélioration de la formation des enseignants qui constitue la stratégie privilégiée afin de réaliser ce projet éducatif.

À l'origine du système, le caractère obligatoire de l'éducation primaire, institué dès 1884 par la Loi d'éducation commune, eut une valeur symbolique de démocratie éducative dont l'enseignement fut le meilleur garant. Les écoles normales ${ }^{1}$ formèrent des générations de maîtresses et de maîtres avec la mission de consolider la nation à travers l'alphabétisation universelle, instrument majeur d'homogénéisation culturelle et d'intégration des immigrants européens qui arrivèrent en Argentine du fait des politiques de peuplement des XIX ${ }^{\mathrm{e}}$ et $\mathrm{XX}^{\mathrm{e}}$ siècles. Des chercheurs argentins comme Alliaud, Birgin, Davini, Pineau, Puiggrós s'accordent à reconnaître le poids du normalisme dans le processus de construction de la nation, sous l'influence des démocraties libérales.

L'enseignement secondaire et la formation qui y est liée firent naître d'autres pratiques et d'autres conceptions. En effet, depuis le début du $\mathrm{XX}^{\mathrm{e}}$ siècle, les débats sur la légitimité requise afin d'exercer l'enseignement dans le secondaire révélaient les intérêts à l'origine de chaque modalité de ce niveau. Les établissements du secondaire furent initialement « des collèges sélectifs et discriminants » (Tiramoni et Siegler, 2008) qui s'occupaient de l'éducation des élites en recourant à des professeurs de formation libérale dont la plupart avaient été recrutés directement en Europe.

Plus tard, les modalités du cycle secondaire se diversifièrent avec la naissance d'écoles commerciales et techniques qui répondaient aux besoins de formation des classes moyennes alors en pleine expansion. Toutefois, à qui devait incomber les tâches d'enseignement dans ces écoles qui furent méritocratiques dès leur apparition ? À des avocats, à des médecins, à des ingénieurs, à des

\footnotetext{
* Article traduit par Philippe Rabaté.

1. C'est en 1870 qu'est fondée l'École normale de Paraná. Selon Diker et Terigi (1997), cette création signifia l'institutionnalisation du « normalisme » en Argentine. Bien qu'il existât déjà des écoles normales dans certaines provinces, les auteures soutiennent que l'École normale de Paraná fonctionna « comme le modèle sur lequel devaient se fonder toutes les écoles normales du pays ».
} 
comptables qui, outre la profession qu'ils exerçaient déjà, s’impliquaient dans les salles de classe ? Ou bien à des enseignants formés dans des filières professorales spécialisées dans une discipline particulière?

Bien que la formation des enseignants pour le niveau secondaire continue à susciter des débats, le besoin de formation pédagogique est communément accepté et, surtout, prescrit par les statuts qui encadrent le travail enseignant. Les discussions actuelles portent plus précisément sur le poids relatif du disciplinaire et du pédagogico-didactique dans les plans d'étude.

Dans les années soixante, les conceptions technicistes s'imposèrent et produisirent le changement le plus important qu'ait jamais connu le système depuis sa naissance : la tertiarisation de la formation des enseignants, décrétée par le gouvernement militaire issu du coup d'État de 1966.

En 1983, après la dernière dictature militaire, l'Argentine retrouva la vie démocratique. L'enseignement commença alors à être conçu comme un levier stratégique dans la construction d'une citoyenneté active, dans la défense de l'ordre constitutionnel et des droits de l'homme.

Les années d'autoritarisme avaient toutefois laissé leurs marques sur la vie scolaire et, par conséquent, sur l'apprentissage de la profession. Cette réalité impliqua de définir des programmes de formation continue susceptibles de promouvoir la réappropriation de la parole et de la pensée de la part des maîtres et professeurs. Comment mener cette entreprise à bien si les formateurs avaient également subi ce terrible traumatisme, si la production théorique nationale avait été perdue $?^{2}$

Le retour de la démocratie eut une autre conséquence dans la formation des enseignants : la mise en place d'une spécialisation pour l'exercice de l'enseignement dans le niveau supérieur et l'apparition, dans le pays, du champ de la pédagogie de la formation. Sa plus grande expansion eut lieu dans le domaine universitaire, avec le développement des études de troisième cycle, des licences et des études professorales destinées à la formation des formateurs. Les recherches nationales, les expériences pratiques et les débats conceptuels furent à l'origine d'une intéressante production éditoriale d'auteurs argentins sur les grands thèmes de la pédagogie de la formation.

Dans les années 1990, à partir de la réforme éducative issue de la Loi fédérale d'éducation, la structure du système éducatif se modifia et la formation des enseignants également. Ce bouleversement fut accompagné par un processus de transformation curriculaire qui s'appliqua à tous les niveaux et à toutes les modalités. On lança au même moment un «méga » programme de formation continue basé sur une hypothèse de manques selon laquelle la formation initiale hypothéquait la possibilité que les enseignants puissent enseigner les nouveaux

2. Entre 1984 et 1989, les projets de l'École de formation, de perfectionnement et de remise à jour des enseignants de ce qui était alors la municipalité de Buenos Aires avaient comme principal objectif de faire des écoles « un laboratoire de vie démocratique $»$. 
contenus. Ainsi, avec l'appui financier du ministère national, des formations avec des thématiques préétablies, organisées en parcours, furent instituées.

Ce modèle alternatif de formation, centré sur les compétences individuelles de l'enseignant, donna un élan considérable à la formation continue, avec une massification des destinataires mais aussi des formateurs qui en étaient chargés. Son caractère technocratique et ses processus de gestion (à la charge des institutions publiques et privées, reconnues par le réseau fédéral de formation continue des enseignants, que l'État supervisait et auquel il versait les fonds) furent amplement critiqués et inspirèrent - à rebours - des programmes postérieurs centrés sur les écoles, les équipes enseignantes et les problèmes de rendement des élèves.

Nous avons donc présenté synthétiquement certains antécédents des politiques actuelles de transformation du système de formation, afin de donner un cadre à ses orientations fondamentales dans la mesure où celles-ci surgissent naturellement de l'analyse critique de ces mêmes antécédents ${ }^{3}$.

\section{L'ORGANISATION ET LA GESTION DE LA FORMATION DES ENSEIGNANTS}

L'Argentine étant une république fédérale, organisée politiquement en vingt-trois États provinciaux auxquels s'ajoute la ville autonome de Buenos Aires, le gouvernement de l'éducation incombe aussi bien à l'État central - à travers le ministère de l'éducation - qu'aux ministères de l'éducation des différentes provinces.

Le Conseil fédéral d'éducation (CFE) est l'organisme responsable de la coordination des politiques éducatives. Il est présidé par un représentant du ministère au niveau national et y siègent les différents ministères provinciaux ainsi que les représentants des universités.

Dans chaque état provincial et dans la ville autonome de Buenos Aires, la DES (Direction de l'enseignement supérieur) prend en charge la formation des enseignants.

Au niveau des institutions, les ISFD (instituts supérieurs de formation des enseignants) sont gérés par une équipe de direction qui est responsable de l'institution formatrice à la fois sur le plan pédagogique et administratif. Dans certaines provinces, un conseil consultatif peut également exister; il est alors constitué par les coordinateurs des filières, ainsi que par les représentants des étudiants, des professeurs et des diplômés.

En 2006 fut promulguée la LEN (Loi d'éducation nationale). L'on entendait ainsi répondre aux demandes pressantes de différents secteurs pour consolider les responsabilités de l'État national et des États provinciaux afin de garantir à tous les habitants le droit à l'éducation. 
La LEN établit que la formation des enseignants est un instrument privilégié pour la réalisation des politiques éducatives, et qu'elle a pour rôle de fournir au pays des instituteurs et des professeurs capables de transmettre et de créer des connaissances, d'influer sur la formation des personnes, sur le développement national et sur la construction d'une société plus juste.

À partir de cette norme légale, l'INFD (Institut national de formation des enseignants) fut créé et commença à fonctionner en avril 2007. L'INFD fait partie intégrante de la gestion de la formation des professeurs du pays puisque, par l'intermédiaire de sa direction exécutive, des directions nationales de développement institutionnel, de développement professionnel et de recherche ainsi que par le biais du Conseil fédéral - auquel prennent part les membres titulaires des DES -, il planifie et met en œuvre les politiques d'articulation entre la formation initiale et la formation continue.

Il y a dans le pays un peu moins de 1100 ISFD, et $53 \%$ d'entre eux sont gérés étatiquement, financés intégralement par l'État et gratuits. Les institutions de gestion privée sont obligées, afin de recevoir un financement étatique, d'offrir la gratuité des services prévus dans le plan d'études ${ }^{4}$.

Dans de nombreuses localités petites et très éloignées des principaux centres urbains des provinces, les ISFD constituent l'unique offre éducative d'enseignement supérieur. Dans les petits villages, les communautés valorisent l'existence de filières professorales parce que les jeunes y trouvent une possibilité de suivre des études alors même que leurs conditions de vie ne leur permettent pas de rechercher des formations loin de leurs foyers.

Les ISFD délivrent des diplômes de professeurs qui ont une validité nationale s'ils sont reconnus par les accords fédéraux. Il existe des filières professorales d'éducation initiale, d'éducation primaire, d'éducation secondaire, d'éducation spéciale, d'éducation interculturelle bilingue.

Les filières professorales de disciplines artistiques délivrent des diplômes qui habilitent les étudiants à enseigner dans l'ensemble des niveaux, de la même façon que pour ceux d'éducation physique, de technologie, de langues étrangères ou de peuples indigènes.

Les filières professorales pour l'éducation secondaire forment des étudiants pour chaque discipline de ce niveau (histoire, géographie, etc.) et offrent une formation générale (qui inclut la formation pédagogique et didactique), une formation disciplinaire et une formation pratique depuis le début du cursus. Certaines de ces filières préparent des enseignants pour le supérieur.

4. Il est important de préciser que, parmi les institutions de gestion publique et de gestion privée, il existe une différence importante dans les conditions d'accès à la charge d'enseignant pour tous les niveaux éducatifs. Dans les premières, les antécédents académiques et la trajectoire (le nombre d'années de travail) définissent un rang de classement à partir duquel sont appelés les enseignants. En revanche, les institutions privées peuvent recruter une personne à la seule condition qu'il ait obtenu le diplôme requis par l'État. 
Les universités organisent également des cycles d'études professorales qui touchent des disciplines spécifiques à l'éducation secondaire et à l'enseignement supérieur. Ceux-ci comportent des plans d'étude qui contiennent des matières communes à ces différentes filières professorales, aux licences ainsi qu'à des pans de formation pédagogique (constitués par des disciplines de type recherche) qui sont suivies tout au long du cycle. La partie finale est destinée aux pratiques d'enseignement. La logique de cette formation est fortement centrée sur le savoir disciplinaire car la période de stage est d'ordinaire très brève (dans certains cas, elle ne dépasse pas dix heures).

$\mathrm{Au}$ cours de certaines périodes, lorsque les provinces ne parviennent pas à couvrir l'ensemble des postes vacants dans les établissements du secondaires avec des professeurs titulaires, les DES offrent une formation pédagogique, exclusivement valable dans les provinces, aux professionnels qui ne possèdent pas de diplôme d'enseignant.

\section{LES POLITIQUES DE FORMATION DES ENSEIGNANTS}

Comme nous avons pu le signaler, les politiques actuelles de formation des enseignants sont liées aux politiques d'amélioration et de démocratisation de l'école argentine. En ce sens, elles se réapproprient une grande partie de l'expérience accumulée depuis le retour de la démocratie et posent de nouvelles orientations. Trois d'entre elles sont fondamentales :

- l'élargissement des missions que l'on assigne au système de formation ;

- l'allongement de la période de formation pour l'exercice de l'enseignement dans le préscolaire et dans le primaire ;

- les changements apportés aux curricula des études professorales.

\section{Les nouvelles missions du système de formation}

La résolution 30/07 du CFE définit dix missions pour le système de formation :

- la formation initiale ;

- l'accompagnement des enseignants débutants ;

- la mise à jour des connaissances disciplinaires et pédagogiques des instituteurs et des professeurs;

- la formation pédagogique des techniciens et des professionnels de différentes spécialités qui souhaiteraient exercer le métier d'enseignant;

- le suivi pédagogique des écoles;

- la formation pour le développement d'activités éducatives dans les institutions non scolaires (centres de loisir, centres culturels, contextes d'enfermement);

- le développement de matériels didactiques; 
- la formation à la prise en charge de différentes fonctions dans le système éducatif ;

- la formation aux fonction de direction et de supervision;

- la recherche.

Tous les ISFD doivent offrir une formation initiale ; en revanche, toutes les autres fonctions - à l'exception de la recherche - sont assignées à quelques institutions formatrices en accord avec une planification globale qui doit répondre aux besoins locaux des systèmes éducatifs.

Comme on aura pu l'observer, la majeure partie de ces fonctions est directement liée au développement professionnel des enseignants : l'on tend en effet à ce que le système de formation approfondisse ses relations avec les autres institutions.

Pour mettre en place cette politique de manière efficiente, d'autres transformations sont nécessaires : par exemple, il paraît indispensable de réorganiser l'ensemble des normes statutaires qui fixent les conditions de service des enseignants et des formateurs, afin qu'ils puissent disposer de temps et d'espaces institutionnels consacrés aux tâches liées à ces fonctions. D'autre part, la mise à jour permanente des formateurs pour l'exercice de ces nouvelles fonctions est absolument fondamentale parce que le véritable défi de la formation continue repose dans la valeur des savoirs qu'elle met en jeu. En ce sens, c'est à l'INFD que sont mises en place les grandes lignes du développement professionnel des formateurs, processus dans lequel s'impliquent des spécialistes nationaux et internationaux :

- développement professionnel des formateurs pour l'accompagnement des enseignants débutants ;

- cycles de développement professionnel du personnel de direction des ISFD ;

- cycles de développement professionnel des formateurs chargés des premiers apprentissages en lecture et écriture (qui souffraient d'une présence faible, voire inexistante, dans les anciennes orientations curriculaires de la formation);

- pour chaque province, montage d'un projet d'appui aux enseignants et à leurs établissements, conçu à partir des problématiques d'enseignement les plus pressantes de chaque province;

- visites d'étude à l'extérieur liées aux didactiques des disciplines, à l'enseignement interculturel et bilingue, à la gestion institutionnelle ainsi qu'aux méthodes d'alphabétisation telles qu'elles sont enseignées dans les structures de formation ;

- études de troisième cycle et stages à la charge des universités nationales et organisés à partir des demandes des différents États provinciaux ;

- formation et accompagnement de tous les ISFD pour l'usage des TICE ;

- installation dans quelques ISFD de centres de mise à jour et d'innovation éducative destinés à travailler avec les écoles de leur environnement. 
En Argentine, la recherche en éducation ne fut menée, de manière hégémonique, que dans les universités. Dans les années 1990, cette fonction fut assignée aux ISFD mais elle connut un faible développement car, dans la majorité des provinces, l'organisation du travail des formateurs ne connut pas la moindre modification. Ceci eut pour conséquence une pratique sporadique de la recherche, et affecta par là même la formation requise pour aborder des programmes importants.

La recherche dans les ISFD connaît actuellement un élan important dans les thématiques liées à l'enseignement, au travail et à la formation des enseignants. Le Département du développement professionnel et de recherche de l'INFD lance ainsi le programme "Connaître pour influer sur les pratiques ", en invitant chaque année les institutions formatrices à présenter des projets de recherche pédagogique afin qu'ils soient évalués et, par la suite, financés.

Bien que les programmes du Département de recherche de l'INFD représentent une stimulation importante et indéniable, le problème de l'organisation du travail académique n'en demeure pas moins réel. Dans les filières professorales, il n'y a pas d'alternance entre investigation et recherche comparable à celle qui existe dans les institutions universitaires.

\section{L'EXTENSION DE LA FORMATION DES ENSEIGNANTS AU PRÉSCOLAIRE ET AU PRIMAIRE}

Depuis le début de l'institutionnalisation de la formation des enseignants, la valeur matérielle (traduite en salaire) et symbolique (prestige social, professionnalisation) des diplômes d'enseignement a dépendu directement de la quantité d'années d'études que ceux-ci exigeaient et de la place que l'on a accordé à la connaissance et à sa transmission dans chaque niveau éducatif.

Comme le souligne Aillaud (1993), «aux origines du normalisme argentin, la méthodologie de l'enseignement acquit une importance décisive face au savoir général ». C’est ainsi que débuta le débat qui devait traverser toute l'histoire de la formation des enseignants : la relation entre théorie et pratique, et le poids respectif de ces dernières dans les plans d'études.

La formation des professeurs en tant que filière destinée au niveau secondaire exista jusqu'au décret du gouvernement militaire de 1969. On décida que, en tant que filière de niveau supérieur, elle devait comporter deux ans et demi d'études, avec une première année consacrée à des disciplines théoriques, une seconde année aux disciplines pratiques et cent heures de stage intensif à la fin du cycle d'études.

Le faible impact de cette formation - dominée par l'idée d'application sur la qualité des processus éducatifs donna lieu à la mise en place de projets au niveau des institutions, des provinces ou de l'État fédéral qui produisirent une 
fragmentation du système de formation non seulement dans le pays mais également à l'intérieur de chaque province.

C'est à cette réalité que la réforme de la formation des enseignants des années 1990 tenta d'offrir une réponse; elle porta à trois ans la durée de la formation des enseignants pour l'éducation initiale et pour les deux premiers cycles de l'éducation générale de base (organisation du système éducatif prescrit par la Loi fédérale d'éducation de 1993, en vigueur jusqu'en 2006).

Avec la réforme de 1993, les possibilités d'amélioration de la formation académique et pratique s'amplifièrent : on incluait en effet, dès le début de ce cycle d'études, des ateliers, séminaires et stages qui permettaient d'exposer des expériences susceptibles d'être abordées en groupe dans des processus d'analyse et de réflexion. Dans certaines provinces toutefois, la définition d'une part significative des espaces curriculaires fut laissée au libre choix de chaque institution. Ceci contribua à la désarticulation du système de formation et affecta la qualité des processus pédagogiques, parce que les formateurs devaient prendre en charge ces espaces sans disposer du temps nécessaire pour se spécialiser dans leurs thématiques.

La formation enseignante pour le niveau préscolaire fut à l'origine une spécialisation destinée aux maîtresses des écoles normales et interdite aux hommes jusqu'à il y a un peu plus d'une vingtaine d'années. La dynamique sociale, avec l'entrée massive des femmes sur le marché du travail, suscita une grande expansion de ce niveau et de la production théorique et pratique sur les processus éducatifs des enfants de 0 à 5 ans, processus qui connurent un développement d'une grande richesse dans l'ensemble du pays.

Dans les deux dernières décennies du $\mathrm{XX}^{\mathrm{e}}$ siècle, la défense du caractère éducatif des institutions préscolaires, qui fut menée par les enseignants, les chercheurs et les formateurs, eut son corrélat curriculaire avec un engouement très marqué pour les contenus d'enseignement. La formation des enseignants intervenant à ce niveau fut également portée à trois ans, avec un poids considérable accordé aux didactiques spéciales.

On reconnaît actuellement que ces deux niveaux - préscolaire et primaire - revêtent une importance stratégique pour assurer une expérience scolaire et des apprentissages susceptibles de préparer les élèves aux autres niveaux : fondamentalement, pour rendre effective l'extension du caractère obligatoire de l'école à l'ensemble du secondaire. La charge d'enseignement, les savoirs requis, la complexité croissante de la société et des connaissances exigent d'amplifier la formation des professeurs. Aussi est-ce pour cette raison qu'en 2006, la LEN, dans son article 75, porte à quatre années la durée du cycle d'études afin d'exercer le métier d'enseignant dans le préscolaire et le primaire.

À partir des dispositions de cette norme légale, le sens et le poids des parcours d'études et de pratique se redéfinissent, et cette situation exige une transformation profonde des curricula de la formation des enseignants. Le défi consiste en ce que cette année complémentaire d'études représente effectivement une année 
supplémentaire de formation et un parcours dans lequel les futurs enseignants puissent accéder à des outils pratiques et conceptuels plus variés et efficaces, à une connaissance directe du territoire scolaire et du métier d'enseignant, à une compréhension des conditions de scolarisation des secteurs les plus défavorisés, qui leur permettent d'agir de manière professionnelle face aux problèmes les plus sérieux que connaissent les écoles argentines (faibles résultats, abandon, redoublement).

Ce souhait exprimé par les instituteurs et par les professeurs du secondaire reçoit l'appui des politiques éducatives par le biais de mesures qui améliorent les conditions matérielles d'enseignement et d'apprentissage : bourses, information sur la mobilité (bulletin étudiant) ou encore mise à disposition de livres, d'ordinateurs et de matériels didactiques pour les écoles et les étudiants. Ceci constitue également un défi pour la formation puisque la mise à profit de ces ressources implique de savoir enseigner en les utilisant.

\section{LE CHANGEMENT CURRICULAIRE DES ÉTUDES PROFESSORALES}

En 2007, les « Grandes lignes curriculaires nationales pour la formation des enseignants du niveau préscolaire » furent approuvées; elles constituent le cadre de référence pour l'élaboration de la norme curriculaire dans chaque province. L'on tente ainsi de créer des conditions globales qui puissent garantir la validité nationale des diplômes.

Tous les cycles d'études pour devenir enseignant devront avoir une durée minimale de quatre ans (soit 2600 heures de cours), avec une marge pour les propositions d'options par les institutions de formation qui ne devra jamais dépasser $20 \%$ de la charge horaire totale.

Les nouvelles grandes lignes de ce programme sont réparties en trois champs de connaissances : formation générale (de type humaniste et culturel) ; formation spécifique pour le niveau éducatif correspondant (les disciplines et leurs didactiques, les politiques éducatives et l'étude des caractéristiques psychologiques, sociales et culturelles des élèves du niveau concerné) ; formation par la pratique professionnelle (intégration progressive dans différents contextes éducatifs et prise en charge graduelle de responsabilités d'enseignement). Avec cette transformation curriculaire, on abandonne l'approche par champs disciplinaires, présente dans les réformes précédentes, pour recentrer la formation autour de disciplines spécifiques.

Ce processus de transformation curriculaire est d'une grande complexité car il doit prendre en considération de nouvelles modalités que la démocratisation et l'universalisation de l'éducation impliquent (ruralité, éducation interculturelle bilingue, éducation dans des contextes d'enfermement) et, par conséquent, il doit également prendre en considération la mise à jour professionnelle des formateurs puisqu'un grand nombre des thèmes impliqués commencent à se développer dans le champ de la pédagogie. 
En ce qui concerne la formation par la pratique professionnelle, l'approche qui sous-tend cette réforme la considère comme l'axe central de la formation, tout en se réappropriant et en amplifiant la conception qui avait inspiré la réforme curriculaire précédente. Elle inclut des activités de terrain (observation institutionnelle, participation à la vie scolaire et communautaire) et des contextes de formation pour l'analyse (ateliers, réunions). Dans ce processus, l'étudiant progresse dans les stages en classe (pratiques de classe), élabore et explore des projets didactiques et achève sa formation par l'étape de résidence au cours de laquelle il prend en charge un cours, avec l'appui de l'enseignant titulaire et des formateurs de l'ISFD.

Cette manière de concevoir l'apprentissage de la pratique professionnelle redéfinit le lien du système de formation avec les établissements scolaires et avec d'autres institutions éducatives. Un intense travail est nécessaire pour que celles-ci ne se bornent pas à recevoir les futurs enseignants mais soient également disposées à organiser pour eux des expériences ayant une véritable valeur formatrice. Ces politiques de développement professionnel peuvent contribuer à la construction d'une professionnalité plus collaborative qui fait des écoles des espaces de travail et d'apprentissage professionnels.

\section{Bibliographie}

ALLIAUD A. (1993) : Los maestros y su historia: Los orígenes del magisterio argentino. Buenos Aires: Centro Editor de América Latina.

BIRGIN A. (1999) : El trabajo de enseñar. Entre la vocación y el mercado: Las nuevas reglas del juego. Buenos Aires: Troque.

CFE. Resolución N³0/07, Annexe I.

DAVINI C. (1995) : La formación docente en cuestión. Política y Pedagogía. Buenos Aires. Paidós.

DIKER G, y TERIGI F. (1997) : La formación de maestros y profesores: Hoja de ruta. Buenos Aires: Paidós.

INFD/MECyT. Lineamientos curriculares nacionales para la formación docente inicial.

INFD/MECyT (2007) : Lineamientos nacionales para la formación docente continua y el desarrollo profesional. Buenos Aires.

TIRAMONTI G., SIEGLER S. (2008) : La educación de las élites. Aspiraciones, estrategias y oportunidades. Buenos Aires. Paidós.

\section{SitographIE}

www.me.gob.ar/

http://cedoc.infd.edu.ar/

http://diniece.gov.ar

http://www.oei.es/bibliotecadigital.php

http:www.abc.gov.ar

http://www.uba.ar 